\title{
Preventing Brain Damage from Hypoxic-Ischemic Encephalopathy in Neonates: Update on Mesenchymal Stromal Cells and Umbilical Cord Blood Cells
}

\author{
Makoto Nabetani, MD, $\mathrm{PhD}^{1}$ Takeo Mukai, MD, PhD ${ }^{2}$ Haruo Shintaku, MD, $\mathrm{PhD}^{3}$ \\ ${ }^{1}$ Department of Pediatrics, Yodogawa Christian Hospital, Osaka, \\ Address for correspondence Makoto Nabetani, MD, PhD, \\ Japan \\ 2 Department of Cell Processing and Transfusion, The Institute of \\ Department of Pediatrics, Yodogawa Christian Hospital, Osaka, Japan, \\ Medical Science, The University of Tokyo, Tokyo, Japan \\ ${ }^{3}$ Department of Pediatrics, Faculty of Medicine, Osaka City \\ 1-7-50 Kunijima, Higashi-yodogawa-ku, Osaka 5330024, Japan \\ (e-mail: a103111@ych.or.jp).
}

University, Osaka, Japan

Am J Perinatol 2022;39:1754-1763.

\section{Abstract}

Keywords

- hypoxic-ischemic encephalopathy

- cerebral palsy

- umbilical cord blood stem cells

- mesenchymal stromal cells

- HIE

- CP

- MSCS

- secondary energy failure

Neonatal hypoxic-ischemic encephalopathy (HIE) causes permanent motor deficit "cerebral palsy (CP)," and may result in significant disability and death. Therapeutic hypothermia $(\mathrm{TH})$ had been established as the first effective therapy for neonates with HIE; however, TH must be initiated within the first 6 hours after birth, and the number needed to treat is from 9 to 11 to prevent brain damage from HIE. Therefore, additional therapies for HIE are highly needed. In this review, we provide an introduction on the mechanisms of HIE cascade and how TH and cell therapies such as umbilical cord blood cells and mesenchymal stromal cells (MSCs), especially umbilical cord-derived MSCs (UC-MSCs), may protect the brain in newborns, and discuss recent progress in regenerative therapies using UC-MSCs for neurological disorders. The brain damage process "HIE cascade" was divided into six stages: (1) energy depletion, (2) impairment of microglia, (3) inflammation, (4) excitotoxity, (5) oxidative stress, and (6) apoptosis in capillary, glia, synapse and/or neuron. The authors showed recent 13 clinical trials using UC-MSCs for neurological disorders. The authors suggest that the next step will include reaching a consensus on cell therapies for HIE and establishment of effective protocols for cell therapy for HIE.
\end{abstract}

\section{Key Points}

- This study includes new insights about cell therapy for neonatal HIE and CP in schema.

- This study shows precise mechanism of neonatal HIE cascade.

- The mechanism of cell therapy by comparing umbilical cord blood stem cell with MSC is shown.

- The review of recent clinical trials of UC-MSC is shown.

received

October 5, 2020

accepted after revision

February 11, 2021

published online

April 14, 2021
DOI https://doi.org/ 10.1055/s-0041-1726451. ISSN 0735-1631.

\footnotetext{
(C) 2021. The Author(s).

This is an open access article published by Thieme under the terms of the Creative Commons Attribution-NonDerivative-NonCommercial-License, permitting copying and reproduction so long as the original work is given appropriate credit. Contents may not be used for commercial purposes, or adapted, remixed, transformed or built upon. (https://creativecommons.org/ licenses/by-nc-nd/4.0/) Thieme Medical Publishers, Inc., 333 Seventh Avenue, 18th Floor, New York, NY 10001, USA
} 
Neonatal mortality rates have decreased considerably over the past several decades, yet the prevalence of severe neurological sequelae such as cerebral palsy (CP), epilepsy, intellectual disability, respiratory disorders, hearing loss, visual disturbances, hydrocephalus, behavioral problems and others due to hypoxic-ischemic encephalopathy (HIE) have remained at a similar rate over several decades. HIE in full-term infants occurs in an estimated 0.5 to 2/1,000 live births and results in severe disability and even death. In recent years, therapeutic hypothermia $(\mathrm{TH})$ has been established as the first effective therapy for neonates with HIE. ${ }^{1}$ However, TH must be initiated within the first 6 hours of birth, and the number needed to treatment is 9 to 11 to prevent brain damage from HIE. Therefore, additional therapies for HIE are highly needed. In this context, cell therapies such as umbilical cord blood stem cells (UCBCs), bone marrow (BM) stem cells, and umbilical cord/BM-derived mesenchymal stromal cells (UC/BM-MSCs) have started to be incorporated into new protocols for protecting against ischemic brain damage. ${ }^{2}$ Interestingly, MSC therapy may also provide promising results for neonates with acute respiratory distress syndrome in the coronavirus disease 2019 infection era. ${ }^{3}$

The pathological characteristics of neonatal brain injury differ markedly from those in adults. For example, energy demand is much smaller hypoxia and brain swelling is not as precarious and there is a secondary energy failure phase due to complicated cascade of HIE. ${ }^{4-6}$ Furthermore, there is a need to overcome "secondary energy failure," since newborns with HIE often deteriorate even after appropriate neonatal cardiopulmonary resuscitation (CPR), respiratory circulation support therapy, and $\mathrm{TH}$.

\section{Mechanism of Neonatal HIE Cascade}

We present the mechanism of the HIE cascade, energy depletion, excitotoxicity, intracellular $\mathrm{Ca}^{2+}$ mobilization, and cell damage process in -Fig. 1.

Olney first reported "excitotoxicity." Meaning that some of the neural cell death due to hypoxia-ischemic (HI) insult was mediated by excess production of the excitatory neurotransmitter "glutamate" and elevation of intracellular $\mathrm{Ca}^{2+}$ concentration by $\mathrm{N}$-methyl-D-aspartate glutamatergic receptor (NMDA GluR), AMPA/Kainate GluR (A/K GluR), and metabolic GluR(mGluR) in different ways. ${ }^{7,8}$ We summarize the mechanism of "excitotoxicity" in - Fig. 1. Glutamate (Glu) is converted to Glutamine (Gly) by the action of glutamine synthetase (GS) in astrocyte, and shuttled from astrocyte to neurons, then converted to Glu by glutaminases (GLS). Energy depletion in presynaptic site of neurons activates release of Glu into synapse. A large proportion of the Glu released at the synapse is taken up by astrocytes via excitatory amino acid transporter together with three $\mathrm{Na}^{+}$ions. This $\mathrm{Na}^{+}$is extruded by the action of the $\mathrm{Na}^{+} / \mathrm{K}+$ ATPase. Glu uptake cannot work enough in the condition of adenosine triphosphate (ATP) reduction. Glu at synapse activates NMDA GluR, A/K GluR, and mGluR.

In contrast to the experience with adult $\mathrm{HI}$ insults, some newborns who had recovered from severe asphyxia subsequently deteriorated rapidly and expired a few days later. Kirino first reported the phenomenon "Delayed neuronal death." 9 Delpy et al reported the phenomenon of delayed neuronal death in newborns after HI insults, termed "secondary energy failure," using a phosphorus magnetic resonance spectroscopy to replicate the complicated process in piglets and rat pups in the 1990 s. $^{10-13}$ Simon et al proposed that brain damage due to HIE can be treated by NMDA antagonists and suggested that brain damage due to HIE could be blocked pharmacologically to protect against neonatal HIE. ${ }^{14}$ Unfortunately, NMDA receptor blocker and other drugs, such as calcium channel antagonists and magnesium sulfate, were not effective in clinical care. We reported that irreversible neuronal cell damage was triggered by an elevation of intracellular $\mathrm{Ca}^{2+}$ concentration subsequent to excessive accumulation of the excitatory neurotransmitter glutamate in immature and mature rats during ischemia and glucose deprivation. ${ }^{15}$ Furthermore, there is increasing evidence that mitochondrial dysfunction generated by excessive intracellular $\mathrm{Ca}^{2+}$ accumulation results in oxidative stress, apoptosis, and necrosis. We summarize the mechanism of elevation of intracellular $\mathrm{Ca}^{2+}$ concentration and irreversible neuronal cell death in - Fig. 1. Calcium influx by NMDA GluR directly increases intracellular $\mathrm{Ca}^{2+}$ concentration. A/K receptors flux large amounts of sodium, depolarizing cell membrane, and blocking $\mathrm{Ca}^{2+}$ efflux from neurons by Cation/ $\mathrm{Ca}^{2+}$ antiporter. Depolarization of cell membrane activates voltage-sensitive $\mathrm{Ca}$ channels and facilitates NMDA GluR activation. Signaling of mGluR by Glu, activating phospholipase C (PLC), facilitates inositol 1,4,5-triphosphate (IP3) and activates IP3 inducing calcium release from endoplasmic reticulum (ER). Therefore, activation of three different GluRs leads to elevation of intracellular $\mathrm{Ca}^{2+}$ concentration in different ways. Furthermore, elevation of intracellular $\mathrm{Ca}^{2+}$ concentration activates calcium-induced calcium release from ER. $\mathrm{Ca}^{2+}$ efflux from neurons by $\mathrm{Ca}^{2+}$ ATPase cannot work enough to prevent elevation of intracellular $\mathrm{Ca}^{2+}$ concentration in the condition of ATP deduction. These multiple mechanisms of HIE induce steep elevation of intracellular $\mathrm{Ca}^{2+}$ concentration from $10^{-7} \mathrm{M}$ in the condition of ATP reduction and reach plateau level in several minutes as we show in our article. Elevation of intracellular $\mathrm{Ca}^{2+}$ concentration deteriorates mitochondrial function and leads to accumulations of free radicals, necrosis, and apoptosis. After plateau level of intracellular $\mathrm{Ca}^{2+}$ concentration, neuronal damage becomes irreversible. So, recent therapies in the acute phase of HIE could be expected with the aim of suppressing the commencement of brain damage cascade.

On the contrary, we reported that the presence of glucose is essential for neural activity in the adult rat and showed that glucose metabolites such as lactate and $\beta$-hydroxybutyrate (OHBA) are available for both neural activity and maintaining the level of high-energy phosphates in a tissue of hippocampus slice in the immature rats. ${ }^{16}$ We also reported the possibility of lactate preserving neural function of the adult brain. ${ }^{17}$ We further described the relationship between neural activity and the levels of high energy phosphates during deprivation of oxygen and/or glucose in hippocampal slices of immature and adult rats. Our results indicate that the immature rat is extremely resistant to 


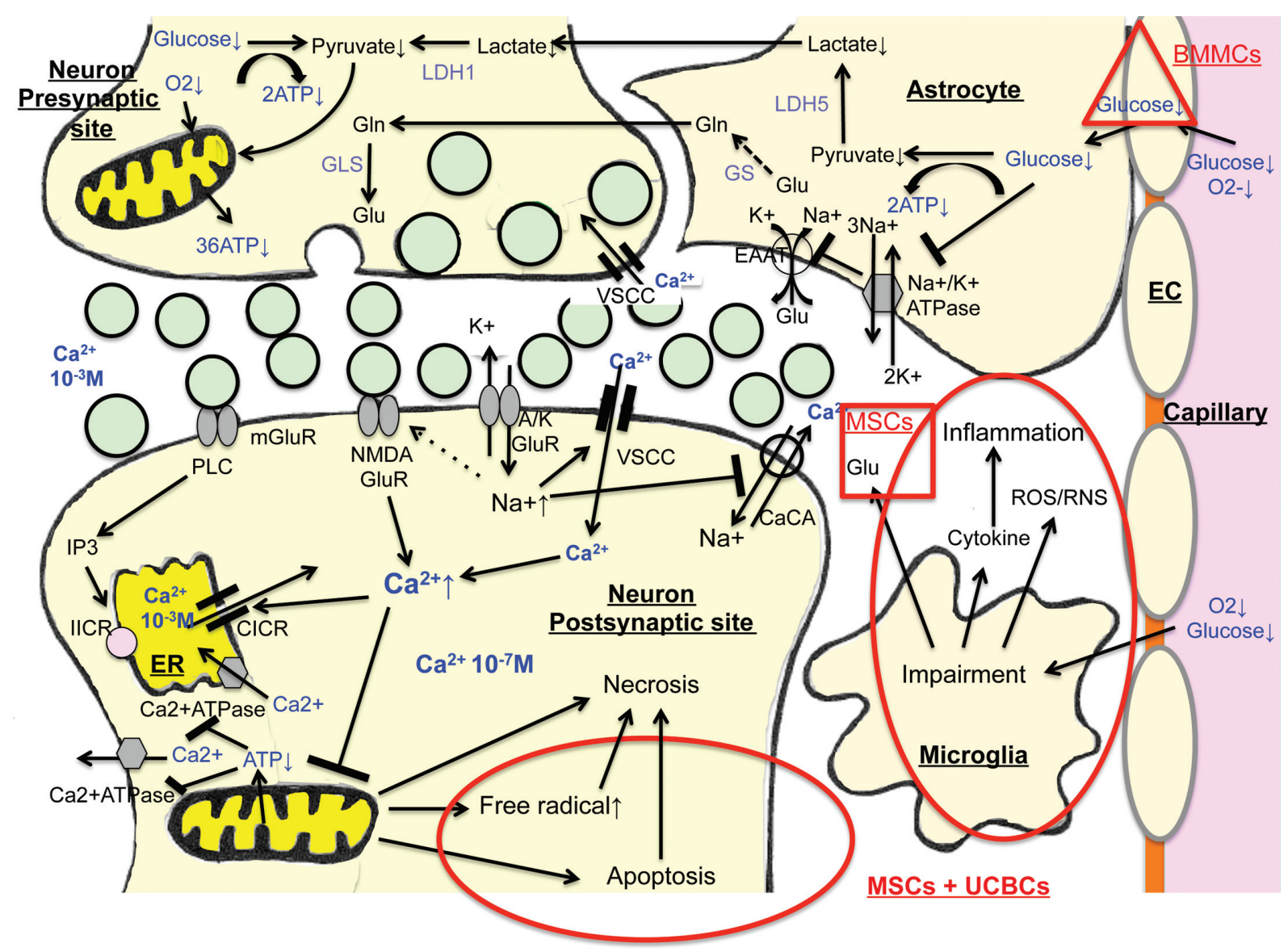

Fig. 1 Mechanism of hypoxic-ischemic encephalopathy (HIE) cascade and cell therapy for HIE cascade. First, HIE induces energy depletion (O2-, Glucose-) in a capillary and glucose reduction in endothelial cell (EC) and in astrocyte. Glucose reduction in astrocyte leads to reduction of pyruvate and lactate that is converted from pyruvate by lactate dehydrogenase 5 (LDH5). And it leads to reduction of lactate and reduction of pyruvate that is converted from lactate-by-lactate dehydrogenase $1(\mathrm{LDH} 1)$ in presynaptic site. Then, pyruvate reduction with $\mathrm{O} 2$ reduction leads to adenosine triphosphate (ATP) reduction in mitochondria. Glutamate (Glu) is converted to glutamine (Gly) by the action of glutamine synthetase (GS) in astrocyte, and shuttled from astrocyte to neurons, then converted to Glu by glutaminases (GLS). Energy depletion in presynaptic site of neurons activates release of Glu into synapse. A large proportion of the Glu released at the synapse is taken up by astrocytes via excitatory amino acid transporter (EAAT) together with three $\mathrm{Na}^{+}$ions. This $\mathrm{Na}^{+}$is extruded by the action of the $\mathrm{Na}^{+} / \mathrm{K}+\mathrm{ATPase}$. Glu uptake cannot work enough in the condition of ATP reduction. ${ }^{83} \mathrm{Glu}$ at synapse activate N-methyl-D-aspartate glutamate glutamatergic receptor (NMDA GluR), AMPA/Kainate glutamatergic receptor (A/K GluR), and metabolic glutamatergic receptor (mGluR). On the other hand, A/K receptors do not directly allow entry of sufficient calcium to increase intracellular $\mathrm{Ca}^{2+}$ concentration. However, $\mathrm{A} / \mathrm{K}$ receptors flux large amounts of sodium, depolarizing cell membrane and blocking $\mathrm{Ca}^{2+}$ efflux from neurons by cation/ $\mathrm{Ca}^{2+}$ antiporter (CaCA). Depolarization of cell membrane activates voltage-sensitive Ca channels (VSCC) and facilitate NMDA GluR activation. Signaling of mGluR by Glu, activating phospholipase C (PLC), facilitate inositol 1,4,5-triphosphate (IP3) and activate IP3 induces calcium release (IICR) from endoplasmic reticulum (ER). Furthermore, elevation of intracellular $\mathrm{Ca}^{2+}$ concentration activates calcium-induced calcium release (CICR) from ER. Ca ${ }^{2+}$ efflux from neurons by $\mathrm{Ca}^{2+}$-ATPase cannot work enough to prevent elevation of intracellular $\mathrm{Ca}^{2+}$ concentration in the condition of ATP deduction. These multiple mechanisms of HIE induce steep elevation of intracellular $\mathrm{Ca}^{2+}$ concentration from $10^{-7} \mathrm{M}$ and reach plateau level in several minutes as we show in our article. After plateau level of intracellular $\mathrm{Ca}^{2+}$ concentration, neuronal damage becomes irreversible. ${ }^{15}$ Microglia also play an important role of neuronal cell damage in case of HIE. Energy depletion in a capillary induces impairment of microglia and it facilitates cytokines, Glu, reactive oxygen species (ROS), and reactive nitrogen species (RNS). Red circles show protective effects of mesenchymal stem cell (MSC) and umbilical cord blood stem cells (UCBCs) on impairment of microglia, inflammation, oxidative stress (free radicals and ROS/RNS) and apoptosis. Red square shows a protective effect of MSC on excitotoxicity. Red triangle shows a protective effect of bone marrow mononuclear cells (BM-MNCs) on energy reduction in EC via gap junction. ${ }^{55}$

oxygen deprivation from a functional and metabolic perspectives, whereas in the adult rat, preservation of neural activity highly depends on both oxygen and glucose and that glucose plays an important role in the preservation of neural activity in addition to its major function as an energy substrate, especially in immature animals. ${ }^{6}$ Therapies targeting energy substrates are still the focus of current research. ${ }^{18}$

Ferriero explained that brain damage in the HIE cascade is divided into five stages: (1) energy depletion, (2) inflammation,
(3) excitotoxity, (4) oxidative stress, and (5) apoptosis. ${ }^{19-21}$ Recent reports not only focus on microglia that have a role in immunomodulation but also promote local network synchronization in the synapses in the developing brain. ${ }^{22}$ Impairment of microglia plays a key role in most early stages of HIE cascade, especially in terms of inflammation and that may continue during a period of days to weeks. We summarize the mechanism of impairment of microglia in case of HIE in - Fig. 1. Energy depletion (O2-, Glucose-) in a capillary induces impairment of 


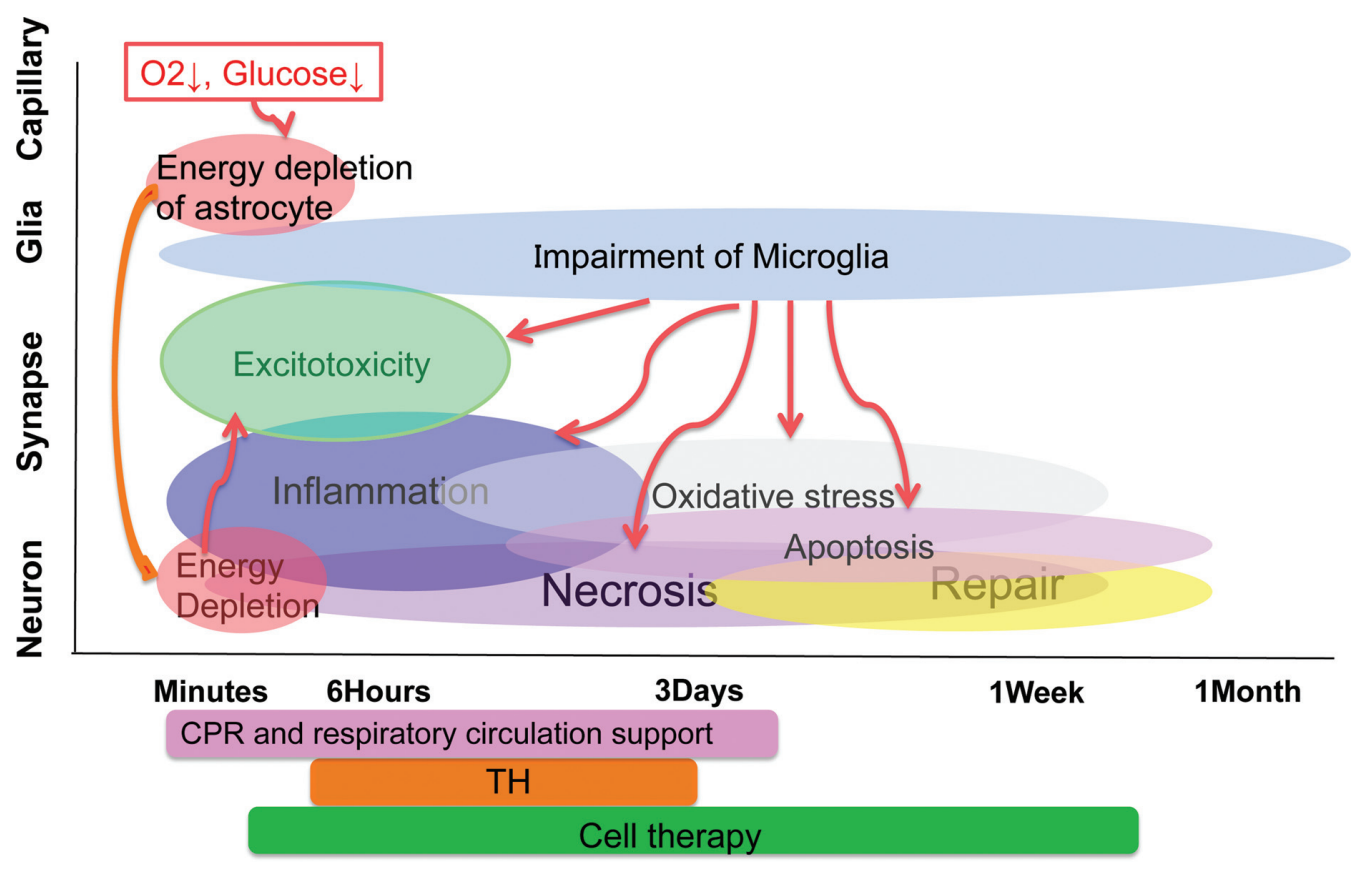

Fig. 2 Timing of promising cell therapies with standard therapies for hypoxic-ischemic encephalopathy cascade. Inflammation, oxidative stress, apoptosis, and necrosis occur through downstream energy depletion, excitotoxicity, and/or impairment of microglia. Cell damage begins immediately after hypoxic-ischemic insult and repair process begin after that. Impairment of microglia, oxidative stress, and apoptosis continues during a period of days to weeks beyond the phase of "secondary energy failure." Cell therapy could also work for days to weeks after neonatal cardiopulmonary resuscitation (CPR), respiratory circulation support therapy, and therapeutic hypothermia (TH) are over.

microglia and it facilitates cytokines, Glu, reactive oxygen species (ROS), and reactive nitrogen species (RNS). Therefore, we suggest an additional mechanism namely "Impairment of microglia" and divide the brain damage process into six stages: (1) energy depletion, (2) impairment of microglia, (3) inflammation, (4) excitotoxity, (5) oxidative stress, and (6) apoptosis (-Fig. 2). Impairment of microglia, oxidative stress, and apoptosis might continue during a period of days or weeks after CPR and respiratory circulation support, so further strategies are needed for this period of time.

\section{How TH and Cell Therapy Prevent Newborn Brain Damage from HIE?}

In 1989, Busto et al showed that mild hypothermia after HI insult in adult rats reduced the release of neurotransmitters and had a protective effect on hippocampal neuronal injury. ${ }^{23}$ In 1996, Thoresen et al reported the protective effect of hypothermia against brain injury in neonatal rats. ${ }^{24,25}$ In 1997, we demonstrated that hypothermia therapy was an effective treatment for hypoxic or ischemic brain damage in rats by suppressing energy loss and elevation of intracellular $\mathrm{Ca}^{2+}$ concentration. The protective effects of hypothermia (33 and $29^{\circ} \mathrm{C}$ ) on the neuronal activity, intracellular $\mathrm{Ca}^{2+}$ accumulation, and ATP levels during deprivation of oxygen and/or glucose were investigated using guinea pig hippocampal slices. ${ }^{26}$ In 2004, McManus et al reported that neuroprotective effects of hypothermia are mediated through a reduction in nitric oxide and superoxide formation and that this effect is likely to be downstream of NMDA receptor activation. ${ }^{27}$ Recent studies showed that $\mathrm{TH}$ has been suggested to provide a protective effect mainly on (1) energy depletion due to reduction in energy metabolism but also other five stages (2) impairment of microglia, ${ }^{28}$ (3) inflammation, ${ }^{29,30}$ (4) excitotoxity, ${ }^{27}(5)$ oxidative stress, ${ }^{27}$ and (6) apoptosis ${ }^{31}$ (-Table 1 ).

In recent years, cell therapies such as UCBCs, umbilical cord-derived mesenchymal stromal cells (UC-MSCs) and bone marrow-derived mesenchymal stromal cells (BMMSCs) are attracting attention due to their HIE neuroprotective ability.

\begin{tabular}{|c|c|c|c|c|c|c|c|}
\hline & $\begin{array}{l}\text { Energy } \\
\text { depletion }\end{array}$ & $\begin{array}{l}\text { Impairment } \\
\text { of Microglia }\end{array}$ & Inflammation & Excitotoxicity & $\begin{array}{l}\text { Oxidative } \\
\text { stress }\end{array}$ & Apoptosis & $\begin{array}{l}\text { Enhance } \\
\text { regeneration }\end{array}$ \\
\hline $\mathrm{TH}$ & $++^{26}$ & $+{ }^{28}$ & $+{ }^{29,30}$ & $+{ }^{27}$ & $+{ }^{27}$ & $++^{31}$ & \\
\hline UCBCs & $+{ }^{59}$ & $+{ }^{42}$ & $+{ }^{48}$ & & $+{ }^{49}$ & $++^{49}$ & $+{ }^{51,53,57}$ \\
\hline MSCs & & $++^{33,43,44}$ & $++^{39,44-47}$ & $+{ }^{34}$ & $+^{34}$ & $+^{34}$ & $+{ }^{35}$ \\
\hline
\end{tabular}

Abbreviations: HIE, hypoxic-ischemic encephalopathy; MSCs, mesenchymal stem cells; TH, therapeutic hypothermia; UCBCs, umbilical cord blood stem cells. 
UCBCs are suggested to provide a protective effect mainly on impairment of microglia, inflammation, oxidative stress and apoptosis, as well as their ability to enhance regeneration. Red circles in - Fig. 1 show protective effects of UCBCs on impairment of microglia, inflammation, oxidative stress (free radicals and ROS/RNS), and apoptosis. While red triangle in - Fig. 1 shows a protective effect of bone marrow mononuclear cells (BM-MNCs) on energy reduction in endothelial cell via gap junction (55) (- Table 1 ).

MSCs are cells derived from several sources as defined with by the International Society for Cellular Therapy; first, MSCs must be plastic-adherent when maintained in standard culture conditions. Second, MSC must express CD105, CD73, and CD90, and lack expression of CD45, CD34, CD14 or CD11b, CD79 $\alpha$, or CD19 and human leukocyte antigen-DR isotype (HLA-DR) surface molecules. Finally, MSC must differentiate to osteoblasts, adipocytes, and chondroblasts in vitro. ${ }^{32}$ MSCs may provide a protective effect against impairment of microglia, ${ }^{33}$ inflammation, excitotoxity, oxidative stress, and apoptosis ${ }^{34}$ Red circles in -Fig. 1 show protective effects of MSC on impairment of microglia, inflammation, oxidative stress (free radicals and ROS/RNS), and apoptosis. And, red square in - Fig. 1 shows a protective effect of MSC on excitotoxicity ( - Table 1). Furthermore, the paracrine effect by neurotrophic factors has been reported in UC-MSCs on neurological regeneration, showing that UCMSCs-conditioned medium enhances Schwann cell's viability and proliferation via increases in nerve growth factor and brain-derived neurotrophic factor (BDNF) expression. ${ }^{35}$ We also found that UC-MSCs secrete BDNF and hepatocyte growth factor (HGF) attenuating brain injury. ${ }^{34,36}$ We also hope that MSC-derived extracellular vesicles will be therapeutic candidates for a successful clinical translation. ${ }^{37}$

However, some reports propose negative effects of cell therapy. Dalous et al demonstrated that UC-MNCs cannot integrate into the developing brain or promote subsequent repair in most conditions tested, and that the intraperitoneal injection of high amounts of UC-MNCs aggravated WMD and was associated with systemic inflammation. ${ }^{38}$ Furthermore, the timing, dose, route of application of UC-MNCs, and UC-MSCs have not yet been precisely determined. A systematic review and meta-analysis of MSC for HIE demonstrated that there is various delivery routes (intracerebral, intranasal, intravenous, and others), various timing of application after $\mathrm{HI}$ insult ( $\leq 72$ hours, $>72$ hours, multiple doses),various source (BM, UC, placenta, not reported), various origin (Allogeneic, Xenogeneic), and various dose $(\leq 250,000$ cells, $>250,000$ cells $-\leq 500,000$ cells, $>500,000$ cells $-\leq 1,000,000$ cells, $>1,000,000$ cells). ${ }^{39}$ Interestingly, combination of $\mathrm{TH}$ and other therapy might worsen the brain injury. ${ }^{40}$ It is still highly controversial whether cell therapy for perinatal brain injury is effective or not.

\section{Reduction in Impairment of Microglia}

Microglia, immune cells of the central nervous system, continuously survey the microenvironment and respond to brain injury. ${ }^{41}$ Microglia are activated in response to brain injury, and are polarized toward an inflammatory phenotype that enhances the generation of pro-inflammatory media- tors such as interleukin- $1 \beta$ and tumor necrosis factor- $\alpha$. However, they can also be polarized to the anti-inflammatory phenotype via mediators such as arginase 1 and transforming growth factor- $\beta$. Therefore, modulation of the phenotype of the microglia may be a novel therapeutic strategy for the treatment of neurological disorders accompanied by inflammation. Li et al reported that UCBC administration at 12 hours after $\mathrm{HI}$ reduces white matter injury by affecting activated microglia. ${ }^{42}$ Recent experimental studies reported that MSCs affect activated microglia. ${ }^{43}$ We found UC-MSCs could immunomodulate activated microglia and decreased their inflammatory cytokines. Moreover, UC-MSCs could change their phenotypes including morphology and phagocytic ability. Morphological and phagocytotic analyses revealed that lipopolysaccharide stimulation significantly changed microglial morphology to amoeboid in which Factin spread with ruffle formation resulting in reduced phagocytosis of Escherichia coli, while MSC co-culture induced shrinkage and concentration of F-actin to form an actin ring, thereby restoring phagocytosis. ${ }^{44}$ These effects of UC-MSCs that modulate the activated microglia may be a therapeutic potential for the treatment of neurological disorders accompanied by inflammation.

\section{Immunomodulation/Anti-inflammatory Action}

It is not yet known which component of cord blood is most efficacious for treating brain injury-mediated inflammation. Specific cell populations found in cord blood and tissue, such as MSCs and endothelial progenitor cells, have demonstrated potential utility for mitigating the inflammatory process induced by brain injury. Immunosuppressive effects have now become the most popular property of MSCs for clinical use. $^{45}$ Defect of HLA-DR (class II) expression in MSCs can theoretically rescue them from immune recognition by CD4+ T cells. ${ }^{46}$ Moreover, MSCs do not express co-stimulatory surface antigens such as CD80, and CD86, which activate T-cells. ${ }^{47}$ Thus, MSCs escape activated $\mathrm{T}$ cells and exert immunomodulation. MSCs are reported to protect brains against global and local neuroinflammatory cascades triggered by $\mathrm{HI}$ events. ${ }^{39,44}$ However, at times MSCs have both anti- and proinflammatory effects. Indeed, in our study, some UC-MSCs caused inflammatory response in restingsurveying (not activated) microglia, indicating that MSCs should not be administered to healthy brain with no inflammation. ${ }^{44}$ Some reports, however, suggest that UCBC administration reduces white matter injury after $\mathrm{HI}$ insult, via a combination of anti-inflammatory and other actions. ${ }^{48}$

\section{Reduction of Oxidative Stress and Apoptosis}

Hattori et al reported that a single intraperitoneal injection of UCB-derived mononuclear cells 6 hours after an ischemic insult was associated with a transient reduction in the number of apoptosis and oxidative stress marker-positive cells, but it did not induce long-term morphological or functional protection. They suggested that repeated administration or a combination treatment of UCB-derived mononuclear cells may be required to achieve sustained protection. ${ }^{49}$ However, MSCs are reported to be able to alleviate oxidative stress, and to reduce apoptosis. 
We also reported UC-MSC-secreted HGF and BDNF have neuroprotective effects on damaged neurons by reducing the number of neurons displaying signs of apoptosis/necrosis. ${ }^{34}$

\section{Enhancement of the Regenerative Process by Secretion of Various Cytokines}

Human CD34+ cells have been shown to secrete various growth factors such as BDNF, glial cell line-derived neurotrophic factor (GDNF), vascular endothelial growth factor (VEGF), and numerous angiogenic factors, including HGF and insulin-like growth factor- $1 .^{50-53}$ MSCs have also been reported to secrete various neurotrophic factors and growth factors such as BDNF, GDNF, HGF, and VEGF. ${ }^{35}$

\section{Enhancement of the Regenerative Process by Angiogenesis for Better Cerebral Circulation}

In 2004, Taguchi et al reported that after a stroke, CD34+ cells provide a favorable environment for neuronal regeneration, suggesting an essential role of CD34+ cells in directly or indirectly promoting an environment conducive to neovascularization of the ischemic brain. Endothelial progenitor cells have angiogenic and vascular reparative capabilities that make them ideal for neurovascular repair. ${ }^{54,55}$ Such a rich vascular environment, along with the generation of other nurturing neuronal mediators from CD34+ cells, such as VEGF, epidermal growth factor 2, and insulin-like growth factor 1-1, enhances subsequent neuronal regeneration. ${ }^{56,57}$ Endogenous neurogenesis is accelerated by neuronal progenitors to the damaged area, followed by their maturation and survival when CD34+ cells continue to stimulate the formation of vascular channels. ${ }^{58}$ In 2020, Kikuchi-Taura et al reported that angiogenesis is activated by BM-MNCs via gap junction-mediated cell-cell interaction and that cell-cell interaction via gap junction is the prominent pathway for activation of angiogenesis at endothelial cells after ischemia and provided novel paradigm that energy source supply by stem cell to injured cell is one of the therapeutic mechanisms of cell-based therapy. ${ }^{59}$

\section{Enhancement of the Regenerative Process by Neurogenesis}

Neural stem/progenitor cells participate in the regenerative response to perinatal $\mathrm{HI}^{60}$ One report suggests that hematopoietic stem cells could differentiate into nonlymphohematopoietic cells such as neurons or microglia or could stimulate neurogenesis. ${ }^{61}$ However, it is uncertain whether this is significantly effective for neonates with HIE. ${ }^{62-65}$ We reported UC-MSCs could enhance neurogenesis with high expression of growth-associated protein 43 in injured neurons, and also confirmed elongated processes in injured neurons. We also previously showed that UC-MSCs exert their neuroprotective effects partially through secretion of BDNF and HGF by inhibiting the apoptosis/necrosis of injured neurons. ${ }^{34}$

\section{History of Clinical Therapies for HIE}

\section{History of TH and Cell Therapies for Neonatal HIE}

The 2010 revised International Liaison Committee on Resuscitation guidelines ${ }^{66}$ stated that infants born at or near term with evolving moderate-to-severe HIE should be offered TH, based on three large-scale randomized controlled trials.

However, TH must be initiated within the first 6 hours after birth. TH showed protective effects against HIE mainly in acute stages. By contrast, cell therapy may have a much longer therapeutic time window over acute stages because it might reduce apoptosis/oxidative stress and enhance the regenerative process. Furthermore, cell therapies such as UCBCs and UC-MSCs are being incorporated into new protocols for protection against ischemic brain damage in some countries. Cotten et al reported autologous UCBC phase 1 clinical study for newborns with HIE for the first time in $2013 .{ }^{55}$ Twenty-three infants were cooled and received cells. Median collection and infusion volumes were 36 and $4.3 \mathrm{~mL}$. Vital signs including oxygen saturation were similar before and after infusions in the first 48 postnatal hours. Cell recipients and concurrent cooled infants had similar hospital outcomes (mortality, oral feeds at discharge). Thirteen of 18 (74\%) cell recipients and 19 of 46 (41\%) concurrent cooled infants with known 1 year outcomes survived with Bayley III scores $\geq 85$ in three domains (cognitive, language, and motor development). ${ }^{67}$ In 2014, we administered autologous UCBC therapy for neonatal HIE, for the first time in Japan. ${ }^{2}$ In 2014, we established the Neonatal Encephalopathy Consortium, Japan research group for autologous UCBC therapy for neonatal HIE and started using autologous UCBC therapy for neonatal HIE. This is a pilot study for testing the feasibility and safety of UCBC therapy in infants with neonatal HIE; the study is an open-label, single-group assignment. The enrollment criteria for our autologous UCBC study are the same as the inclusion/exclusion criteria for TH in Japan. If a neonate is born with signs and symptoms of moderate-to-severe encephalopathy and meets the criteria for $\mathrm{TH}$, the neonate is considered for entry to this clinical study. There were no serious adverse events that might be related to cell therapy in all six newborns. At 30 days of age, the six infants survived without circulatory or respiratory support. At 18 months of age, neurological development was normal in four infants and delayed in two infants. This study shows that autologous UCBC therapy is feasible and safe. ${ }^{68}$

Recently, MSCs have been attracting much attention for their therapeutic potential for neurological disorders. ${ }^{69}$ Huang et al administered UC-MSCs for CP and reported that UC-MSC infusion with basic rehabilitation was safe and effective in improving gross motor functions in children with $\mathrm{CP}^{70}$ On the contrary, the Nagamura-Mukai group focused on UC because of (1) abundance and ease of collection, (2) noninvasive collection, (3) little ethical controversy, (4) low immunogenicity with significant immunosuppressive ability, and (5) migration ability toward injured sites. ${ }^{36,45}$ We plan to use UC-MSCs for neonatal HIE or CP, after using them as a regenerative product for GVHD.

\section{Clinical Trials Using UC-MSCs}

Clinical trials using UC-MSCs for neurological disorders have been increasing in number and the recent clinical reports are summarized in = Table 2. 


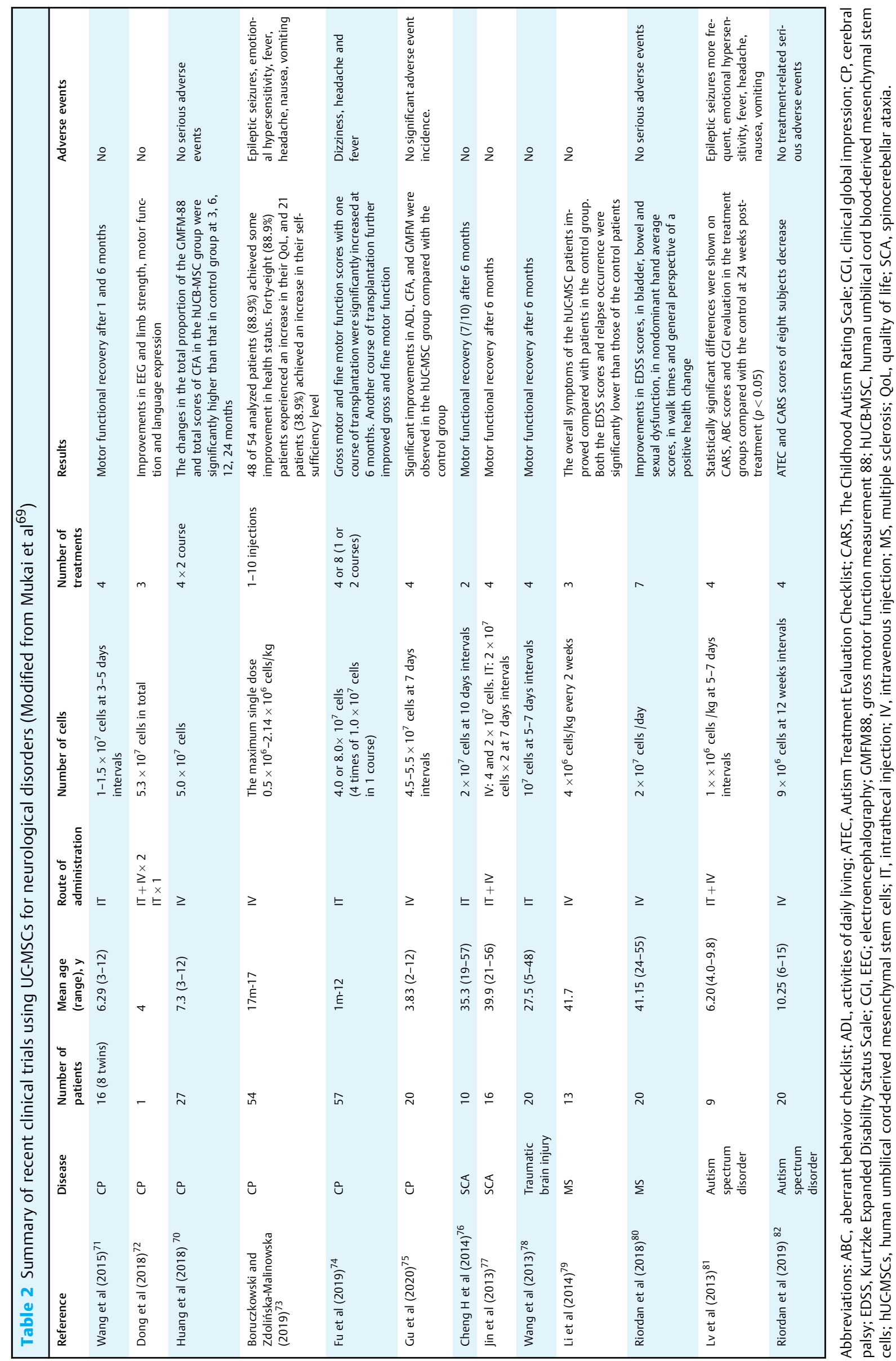


Recent clinical trials using UC-MSCs cover a wide range of neurological diseases including $\mathrm{CP}(n=6)$, spinal cord injury $(n=1)$, spinocerebellar ataxia $(n=1)$, traumatic brain injury $(n=1)$, multiple sclerosis $(n=2)$, and autism spectrum disorder $(n=2)$. Most studies were performed using intrathecal (IT) $(n=5)$, intravenous (IV) $(n=6)$, and IT $+\operatorname{IV}(n=2)$ injection, and multiple administration of cells: two times $(n=1)$, three times $(n=2)$, four times $(n=6)$, seven times $(n=1)$, eight times $(n=1)$, four or eight times $(n=1)$, and others $(n=1)$. The number of cells of administration is various (each dose $9 \times 10^{6}-8 \times 10^{7}$ cells or $0.5-4.0 \times 0^{6}$ cells $/ \mathrm{kg}$ ). IV injection is easier compared with IT, but UC-MSCs are surely distributed to the central nervous system without being trapped in the lung and blood-brain barrier in IT injection

Autologous transplantation is required to avoid transplant rejection, but timing is crucial. UC-MSCs preparation takes 3 months or more and to confirm their quality (from infection and genetic testing), it is impossible to administer autologous UC-MSCs in the acute phase after neurological injuries. Also, establishment of a system in cryopreservation of autologous UC is required. Nonetheless, allogeneic MSCs can be ordered as a preparation anytime and administered in the acute to subacute phase.

Considering UC-MSC therapy for HIE, administration of UC-MSCs in the acute phase might be expected with the aim of suppressing the commencement of brain damage cascade. Therefore, UC-MSCs used for HIE should be allogeneic and should be prepared immediately. UC-MSC therapy for CP is performed considering neurotrophic effects of UC-MSCs in addition to immunomodulation against chronic inflammation. Hence, UC-MSCs used for CP could be both autologous and allogeneic.

Few serious adverse events were observed after transplantation, and most reports suggest that UC-MSC has a therapeutic potential with relative safety. MSCs for neurological diseases are expected as a new cell therapy by combining with rehabilitation and other medication therapies suggested by recent clinical trials.

\section{Conclusion}

Since the establishment of consensus suggesting that $\mathrm{TH}$ should be offered for newborn HIE, it is still challenging to prevent brain damage from complicated cascade of HIE. We suggest that the next step will include reaching a consensus on cell therapies for HIE and establishment of effective protocols for cell therapy for HIE.

\section{Authors' Contributions}

M.N. and H.S conceptualized the manuscript; T.M. was involved in methodology and software; T.M. and H.S. were involved in validation. M.N. wrote the original draft and was involved in formal analysis and investigation, and manuscript preparation, review, and editing. H.S. was involved in supervision, project administration, and funding acquisition. All authors have read and agreed to the published version of the manuscript.
Funding

H.S. received public grant from Japan Agency for Medical Research and Development for autologous UCB stem cell therapy for neonatal HIE in Japan.

\section{Conflict of Interest}

None declared.

\section{Acknowledgments}

The authors thank Dr. Akihiko Taguchi and Prof. Ran D. Goldman for their valuable advice.

\section{References}

1 Jacobs S, Hunt R, Tarnow-Mordi W, Inder T, Davis P. Cooling for newborns with hypoxic ischaemic encephalopathy. Cochrane Database Syst Rev 2007;(04):CD003311

2 Nabetani M, Shintaku H, Hamazaki T. Future perspectives of cell therapy for neonatal hypoxic-ischemic encephalopathy. Pediatr Res 2018;83(1-2):356-363

3 Khoury M, Cuenca J, Cruz FF, Figueroa FE, Rocco PRM, Weiss DJ. Current status of cell-based therapies for respiratory virus infections: applicability to COVID-19. Eur Respir J 2020;55(06): 2000858

4 Perlman JM. Intervention strategies for neonatal hypoxic-ischemic cerebral injury. Clin Ther 2006;28(09):1353-1365

5 Volpe JJ. Hypoxic-Ischemic Encephalopathy: Neuropathology and Pathogenesis. Neurology of the Newborn 5th edition Philadelphia: Saunders; 2008:347-399

6 Nabetani M, Okada Y, Kawai S, Nakamura H. Neural activity and the levels of high energy phosphates during deprivation of oxygen and/or glucose in hippocampal slices of immature and adult rats. Int J Dev Neurosci 1995;13(01):3-12

7 Olney JW, Sharpe LG. Brain lesions in an infant rhesus monkey treated with monosodium glutamate. Science 1969;166 (3903):386-388

8 Olney JW, Ho OL. Brain damage in infant mice following oral intake of glutamate, aspartate or cysteine. Nature 1970;227 (5258):609-611

9 Kirino T. Delayed neuronal death in the gerbil hippocampus following ischemia. Brain Res 1982;239(01):57-69

10 Delpy DT, Gordon RE, Hope PL, et al. Noninvasive investigation of cerebral ischemia by phosphorus nuclear magnetic resonance. Pediatrics 1982;70(02):310-313

11 Hope PL, Costello AM, Cady EB, et al. Cerebral energy metabolism studied with phosphorus NMR spectroscopy in normal and birthasphyxiated infants. Lancet 1984;2(8399):366-370

12 Blumberg RM, Cady EB, Wigglesworth JS, McKenzie JE, Edwards AD. Relation between delayed impairment of cerebral energy metabolism and infarction following transient focal hypoxiaischaemia in the developing brain. Exp Brain Res 1997;113(01): 130-137

13 Lorek A, Takei Y, Cady EB, et al. Delayed ("secondary") cerebral energy failure after acute hypoxia-ischemia in the newborn piglet: continuous 48-hour studies by phosphorus magnetic resonance spectroscopy. Pediatr Res 1994;36(06):699-706

14 Simon RP, Swan JH, Griffiths T, Meldrum BS. Blockade of Nmethyl-D-aspartate receptors may protect against ischemic damage in the brain. Science 1984;226(4676):850-852

15 Nabetani M, Okada Y, Takata T, Takada S, Nakamura H. Neural activity and intracellular Ca2+ mobilization in the CA1 area of hippocampal slices from immature and mature rats during ischemia or glucose deprivation. Brain Res 1997;769(01): 158-162

16 Wada H, Okada Y, Nabetani M, Nakamura H. The effects of lactate and beta-hydroxybutyrate on the energy metabolism and neural 
activity of hippocampal slices from adult and immature rat. Brain Res Dev Brain Res 1997;101(1-2):1-7

17 Saitoh M, Okada Y, Nabetani M. Effect of mannose, fructose and lactate on the preservation of synaptic potentials in hippocampal slices. Neurosci Lett 1994;171(1-2):125-128

18 Cheng J, Korte N, Nortley R, Sethi H, Tang Y, Attwell D. Targeting pericytes for therapeutic approaches to neurological disorders. Acta Neuropathol 2018;136(04):507-523

19 McCord JM. Oxygen-derived free radicals in postischemic tissue injury. N Engl J Med 1985;312(03):159-163

20 Ferriero DM. Neonatal brain injury. N Engl J Med 2004;351(19): 1985-1995

21 Johnston MV, Fatemi A, Wilson MA, Northington F. Treatment advances in neonatal neuroprotection and neurointensive care. Lancet Neurol 2011;10(04):372-382

22 Ikegami A, Haruwaka K, Wake H. Microglia: Lifelong modulator of neural circuits. Neuropathology 2019;39(03):173-180

23 Busto R, Dietrich WD, Globus MY, Ginsberg MD. Postischemic moderate hypothermia inhibits CA1 hippocampal ischemic neuronal injury. Neurosci Lett 1989;101(03):299-304

24 Thoresen M, Bågenholm R, Løberg EM, Apricena F, Kjellmer I. Posthypoxic cooling of neonatal rats provides protection against brain injury. Arch Dis Child Fetal Neonatal Ed 1996;74(01):F3-F9

25 Sirimanne ES, Blumberg RM, Bossano D, et al. The effect of prolonged modification of cerebral temperature on outcome after hypoxic-ischemic brain injury in the infant rat. Pediatr Res 1996; 39(4 Pt 1):591-597

26 Takata T, Nabetani M, Okada Y. Effects of hypothermia on the neuronal activity, [Ca2+]i accumulation and ATP levels during oxygen and/or glucose deprivation in hippocampal slices of guinea pigs. Neurosci Lett 1997;227(01):41-44

27 McManus T, Sadgrove M, Pringle AK, Chad JE, Sundstrom LE. Intraischaemic hypothermia reduces free radical production and protects against ischaemic insults in cultured hippocampal slices. J Neurochem 2004;91(02):327-336

28 Rocha-Ferreira E, Vincent A, Bright S, Peebles DM, Hristova M. The duration of hypothermia affects short-term neuroprotection in a mouse model of neonatal hypoxic ischaemic injury. PLoS One 2018;13(07):e0199890

29 Perrone S, Weiss MD, Proietti F, et al. Identification of a panel of cytokines in neonates with hypoxic ischemic encephalopathy treated with hypothermia. Cytokine 2018;111:119-124

30 Chevin M, Guiraut C, Sébire G. Effect of hypothermia on interleukin-1 receptor antagonist pharmacodynamics in inflammatorysensitized hypoxic-ischemic encephalopathy of term newborns. J Neuroinflammation 2018;15(01):214

31 Zhou T, Lin H, Jiang L, et al. Mild hypothermia protects hippocampal neurons from oxygen-glucose deprivation injury through inhibiting caspase-3 activation. Cryobiology 2018;80:55-61

32 Dominici M, Le Blanc K, Mueller I, et al. Minimal criteria for defining multipotent mesenchymal stromal cells. The International Society for Cellular Therapy position statement. Cytotherapy 2006;8(04):315-317

33 Ding M, Shen Y, Wang P, et al. Exosomes isolated from human umbilical cord mesenchymal stem cells alleviate neuroinflammation and reduce amyloid-beta deposition by modulating microglial activation in Alzheimer's disease. Neurochem Res 2018;43 (11):2165-2177

34 Mukai T, Tojo A, Nagamura-Inoue T. Umbilical cord-derived mesenchymal stromal cells contribute to neuroprotection in neonatal cortical neurons damaged by oxygen-glucose deprivation. Front Neurol 2018;9:466

35 Guo ZY, Sun X, Xu XL, Zhao Q, Peng J, Wang Y. Human umbilical cord mesenchymal stem cells promote peripheral nerve repair via paracrine mechanisms. Neural Regen Res 2015;10(04): 651-658

36 Mukai T, Mori Y, Shimazu T, et al. Intravenous injection of umbilical cord-derived mesenchymal stromal cells attenuates reactive gliosis and hypomyelination in a neonatal intraventricular hemorrhage model. Neuroscience 2017;355:175-187

37 Sung DK, Sung SI, Ahn SY, Chang YS, Park WS. Thrombin preconditioning boosts biogenesis of extracellular vesicles from mesenchymal stem cells and enriches their cargo contents via protease-activated receptor-mediated signaling pathways. Int J Mol Sci 2019;20(12):E2899

38 Dalous J, Pansiot J, Pham H, et al. Use of human umbilical cord blood mononuclear cells to prevent perinatal brain injury: a preclinical study. Stem Cells Dev 2013;22(01):169-179

39 Archambault J, Moreira A, McDaniel D, Winter L, Sun L, Hornsby P. Therapeutic potential of mesenchymal stromal cells for hypoxic ischemic encephalopathy: a systematic review and meta-analysis of preclinical studies. PLoS One 2017;12(12):e0189895

40 Thoresen M. Combining two good treatments makes it worse. Brain Behav Immun 2018;71:7-8

41 Nimmerjahn A, Kirchhoff F, Helmchen F. Resting microglial cells are highly dynamic surveillants of brain parenchyma in vivo. Science 2005;308(5726):1314-1318

42 Li J, Yawno T, Sutherland A, et al. Preterm white matter brain injury is prevented by early administration of umbilical cord blood cells. Exp Neurol 2016;283(pt. A):179-187

$43 \mathrm{Kim}$ S, Kim YE, Hong S, et al. Reactive microglia and astrocytes in neonatal intraventricular hemorrhage model are blocked by mesenchymal stem cells. Glia 2020;68(01):178-192

44 Mukai T, Martino ED, Tsuji S, et al. Umbilical cord tissue-derived mesenchymal stromal cells immunomodulate and restore actin dynamics and phagocytosis of lipopolysaccharide-activated microglia via the PI3K/Akt/Rho GTPase pathway, with lot-to-lot variation. Cell Death Disc 2021;7:46

45 Nagamura-Inoue T, Mukai T. Umbilical cord is a rich source of mesenchymal stromal cells for cell therapy. Curr Stem Cell Res Ther 2016;11(08):634-642

46 Krampera M, Glennie S, Dyson J, et al. Bone marrow mesenchymal stem cells inhibit the response of naive and memory antigen-specific T cells to their cognate peptide. Blood 2003;101(09):3722-3729

47 Weiss ML, Anderson C, Medicetty S, et al. Immune properties of human umbilical cord Wharton's jelly-derived cells. Stem Cells 2008;26(11):2865-2874

48 Aridas JD, McDonald CA, Paton MC, et al. Cord blood mononuclear cells prevent neuronal apoptosis in response to perinatal asphyxia in the newborn lamb. J Physiol 2016;594(05):1421-1435

49 Hattori T, Sato Y, Kondo T, et al. Administration of umbilical cord blood cells transiently decreased hypoxic-ischemic brain injury in neonatal rats. Dev Neurosci 2015;37(02):95-104

50 Yoshihara T, Taguchi A, Matsuyama T, et al. Increase in circulating CD34-positive cells in patients with angiographic evidence of moyamoya-like vessels. J Cereb Blood Flow Metab 2008;28(06): 1086-1089

51 Rosenkranz K, Kumbruch S, Lebermann K, et al. The chemokine SDF-1/CXCL12 contributes to the 'homing' of umbilical cord blood cells to a hypoxic-ischemic lesion in the rat brain. J Neurosci Res 2010;88(06):1223-1233

52 Yasuhara T, Hara K, Maki M, et al. Mannitol facilitates neurotrophic factor up-regulation and behavioural recovery in neonatal hypoxic-ischaemic rats with human umbilical cord blood grafts. J Cell Mol Med 2010;14(04):914-921

53 Majka M, Janowska-Wieczorek A, Ratajczak J, et al. Numerous growth factors, cytokines, and chemokines are secreted by human CD34(+) cells, myeloblasts, erythroblasts, and megakaryoblasts and regulate normal hematopoiesis in an autocrine/paracrine manner. Blood 2001;97(10):3075-3085

54 Taguchi A, Matsuyama T, Moriwaki H, et al. Circulating CD34positive cells provide an index of cerebrovascular function. Circulation 2004;109(24):2972-2975

55 Taguchi A, Soma T, Tanaka H, et al. Administration of CD34+ cells after stroke enhances neurogenesis via angiogenesis in a mouse model. J Clin Invest 2004;114(03):330-338 
56 Nakatomi H, Kuriu T, Okabe S, et al. Regeneration of hippocampal pyramidal neurons after ischemic brain injury by recruitment of endogenous neural progenitors. Cell 2002;110(04):429-441

57 Drago J, Murphy M, Carroll SM, Harvey RP, Bartlett PF. Fibroblast growth factor-mediated proliferation of central nervous system precursors depends on endogenous production of insulin-like growth factor I. Proc Natl Acad Sci U S A 1991;88(06):2199-2203

58 Kasahara Y, Yamahara K, Soma T, et al. Transplantation of hematopoietic stem cells: intra-arterial versus intravenous administration impacts stroke outcomes in a murine model. Transl Res 2016;176:69-80

59 Kikuchi-Taura A, Okinaka Y, Takeuchi Y, et al. Bone marrow mononuclear cells activate angiogenesis via GAP junction-mediated cell-cell interaction. Stroke 2020;51(04):1279-1289

60 Felling RJ, Snyder MJ, Romanko MJ, et al. Neural stem/progenitor cells participate in the regenerative response to perinatal hypoxia/ischemia. J Neurosci 2006;26(16):4359-4369

61 Eglitis MA, Mezey E. Hematopoietic cells differentiate into both microglia and macroglia in the brains of adult mice. Proc Natl Acad Sci U S A 1997;94(08):4080-4085

62 Mezey E, Key S, Vogelsang G, Szalayova I, Lange GD, Crain B. Transplanted bone marrow generates new neurons in human brains. Proc Natl Acad Sci U S A 2003;100(03):1364-1369

63 Li Y, Adomat H, Guns ET, et al. Identification of a hematopoietic cell dedifferentiation-inducing factor. J Cell Physiol 2016;231 (06):1350-1363

64 Chen SH, Wang JJ, Chen $\mathrm{CH}$, et al. Umbilical cord blood-derived CD34+ cells improve outcomes of traumatic brain injury in rats by stimulating angiogenesis and neurogenesis. Cell Transplant 2014; 23(08):959-979

65 Davoust N, Vuaillat C, Cavillon G, et al. Bone marrow CD34+/ $\mathrm{B} 220+$ progenitors target the inflamed brain and display in vitro differentiation potential toward microglia. FASEB J 2006;20(12): 2081-2092

66 Perlman JM, Wyllie J, Kattwinkel J, et al; Neonatal Resuscitation Chapter Collaborators. Part 11: Neonatal resuscitation: 2010 International Consensus on Cardiopulmonary Resuscitation and Emergency Cardiovascular Care Science With Treatment Recommendations. Circulation 2010;122(16, Suppl 2):S516-S538

67 Cotten CM, Murtha AP, Goldberg RN, et al. Feasibility of autologous cord blood cells for infants with hypoxic-ischemic encephalopathy. J Pediatr 2014;164(05):973-979.e1

68 Tsuji M, Sawada M, Watabe S, et al. Autologous cord blood cell therapy for neonatal hypoxic-ischaemic encephalopathy: a pilot study for feasibility and safety. Sci Rep 2020;10(01):4603

69 Mukai T, Tojo A, Nagamura-Inoue T. Mesenchymal stromal cells as a potential therapeutic for neurological disorders. Regen Ther 2018;9:32-37
70 Huang L, Zhang C, Gu J, et al. A randomized, placebo-controlled trial of human umbilical cord blood mesenchymal stem cell infusion for children with cerebral palsy. Cell Transplant 2018; $27(02): 325-334$

71 Wang X, Hu H, Hua R, et al. Effect of umbilical cord mesenchymal stromal cells on motor functions of identical twins with cerebral palsy: pilot study on the correlation of efficacy and hereditary factors. Cytotherapy 2015;17(02):224-231

72 Dong H, Li G, Shang C, et al. Umbilical cord mesenchymal stem cell (UC-MSC) transplantations for cerebral palsy. Am J Transl Res 2018;10(03):901-906

73 Boruczkowski D, Zdolińska-Malinowska I. Wharton's Jelly mesenchymal stem cell administration improves quality of life and self-sufficiency in children with cerebral palsy: results from a retrospective study. Stem Cells Int 2019;2019:7402151

74 Fu X, Hua R, Wang X, et al. Synergistic improvement in children with cerebral palsy who underwent double-course human Wharton's Jelly stem cell transplantation. Stem Cells Int 2019; 2019:7481069-7481069

$75 \mathrm{GuJ}$, Huang L, Zhang C, et al. Therapeutic evidence of umbilical cordderived mesenchymal stem cell transplantation for cerebral palsy: a randomized, controlled trial. Stem Cell Res Ther 2020;11(01):43

76 Cheng H, Liu X, Hua R, et al. Clinical observation of umbilical cord mesenchymal stem cell transplantation in treatment for sequelae of thoracolumbar spinal cord injury. J Transl Med 2014;12:253

77 Jin JL, Liu Z, Lu ZJ, et al. Safety and efficacy of umbilical cord mesenchymal stem cell therapy in hereditary spinocerebellar ataxia. Curr Neurovasc Res 2013;10(01):11-20

78 Wang S, Cheng H, Dai G, et al. Umbilical cord mesenchymal stem cell transplantation significantly improves neurological function in patients with sequelae of traumatic brain injury. Brain Res 2013;1532:76-84

79 Li JF, Zhang DJ, Geng T, et al. The potential of human umbilical cordderived mesenchymal stem cells as a novel cellular therapy for multiple sclerosis. Cell Transplant 2014;23(suppl 1):S113-S122

80 Riordan NH, Morales I, Fernández G, et al. Clinical feasibility of umbilical cord tissue-derived mesenchymal stem cells in the treatment of multiple sclerosis. J Transl Med 2018;16(01):57

81 Lv YT, Zhang Y, Liu M, et al. Transplantation of human cord blood mononuclear cells and umbilical cord-derived mesenchymal stem cells in autism. J Transl Med 2013;11:196

82 Riordan NH, Hincapié ML, Morales I, et al. Allogeneic human umbilical cord mesenchymal stem cells for the treatment of autism spectrum disorder in children: safety profile and effect on cytokine levels. Stem Cells Transl Med 2019;8(10):1008-1016

83 Bélanger M, Allaman I, Magistretti PJ. Brain energy metabolism: focus on astrocyte-neuron metabolic cooperation. Cell Metab 2011;14(06):724-738 